\title{
PROJECTIONS OF GIBBS MEASURES ON SELF-CONFORMAL SETS
}

\author{
CATHERINE BRUCE AND XIONG JIN
}

\begin{abstract}
We show that for Gibbs measures on self-conformal sets in $\mathbb{R}^{d}(d \geq 2)$ satisfying certain minimal assumptions, without requiring any separation condition, the Hausdorff dimension of orthogonal projections to $k$-dimensional subspaces is the same and is equal to the maximum possible value in all directions. As a corollary we show that Falconer's distance set conjecture holds for this class of self-conformal sets satisfying the open set condition.
\end{abstract}

\section{INTRODUCTION}

Let $d \geq k \geq 2$ be integers, let $K \subset \mathbb{R}^{d}$ be Borel or analytic, and let $\Pi_{d, k}$ be the set of orthogonal projections from $\mathbb{R}^{d}$ to its $k$-dimensional subspaces, with natural Haar measure $\xi$. Then

$$
\operatorname{dim}_{H} \pi K=\min \left\{k, \operatorname{dim}_{H} K\right\} \text { for } \xi \text {-almost every } \pi \in \Pi_{d, k} .
$$

This famous result, due to Marstrand [14] in the plane and Mattila [15] in $\mathbb{R}^{d}$, has been the basis for a great deal of work in the field of fractal geometry. Until fairly recently, most of this work concerned general Borel sets $K$ and almost all projections $\pi \in \Pi_{d, k}$. However, Furstenburg's innovative CP-chain method [11] enabled Hochman and Shmerkin [12] to show that for self-similar sets and measures with dense rotations and which satisfy the strong separation condition, the result holds for all $\pi \in \Pi_{d, k}$. Since then, their work has been followed up by many mathematicians, see the recent survey papers [6, 21, 16] and the references therein. In particular, Falconer and Jin [7] extended their result to random cascade measures (including self-similar measures as special cases) without requiring any separation condition.

In [12] Hochman and Shmerkin also considered the projections of products of Gibbs measures on one-dimensional non-linear Cantor sets. The authors used the so-called limit diffeomorphisms of one-dimensional non-linear iterated function systems developed by Sullivan [24] and Bedford and Fisher [1] to transfer the problem back to the affine case. In higher dimensions, Fraser and Pollicott [10] showed that for Gibbs measures on selfconformal sets with the strong separation condition there exists a limit conformal map under which the Gibbs measures generate a CP-chain. But the strong projection theorem for self-conformal measures cannot be directly proved from this result since the dimension of projections is not preserved under conformal maps, and the dense rotations condition is not clear in their setting.

The main difficulty of studying CP-chains/scenery flows of self-conformal measures comes from the fact that linear "zooming-in" operators do not fit well with the non-linear iterated function systems. In this paper we use the methods from Falconer and Jin [7, along with those from Hochman and Shmerkin [12], to overcome this difficulty. The main idea (Lemma 3.4) is first to zoom-in on measures on the symbolic space, or in other words, to zoom-in with conformal mappings, in order to generate a CP-chain, then zoomin on the conditional measures in the CP-chain with linear scale functions to estimate the entropy distortions. We also make clear how to formulate an analogue of the dense 
rotations condition for the minimality of the underlining dynamical system. The methods from [7] also remove the requirement of any separation condition on the underlying sets.

Here we will consider an iterated function system (IFS) of conformal $C^{1+\epsilon}$-maps $\mathcal{I}=$ $\left\{f_{i}\right\}_{i=1}^{m}(m \geq 2)$ in $\mathbb{R}^{d}$ satisfying the following assumptions:

(A0) There is a bounded, convex open set $U \subset \mathbb{R}^{d}$ such that each $f_{i}: U \rightarrow U$ is an injective conformal map, that is $f_{i}(x) \in U$, the derivative $f_{i}^{\prime}(x)$ exists for every $x \in U$ and is a scalar times a rotation matrix, which we may write as

$$
f_{i}^{\prime}(x)=r_{i}(x) O_{i}(x),
$$

where $r_{i}(x) \in(0, \infty)$ and $O_{i}(x) \in S O(d, \mathbb{R})$.

(A1) There exists a constant $0<r^{*}<1$ such that $r_{i}(x) \leq r^{*}$ for every $1 \leq i \leq m$ and $x \in U$.

Remark 1.1. Here we assume the open set $U$ to be convex just for simplicity. It is sufficient to assume that $U$ is connected. The only proof that will be affected by this is Lemma 3.2, where instead of connecting two points in $U$ by a line segment, we can connect two points by smooth curves within $U$, see [18] for example.

These assumptions imply that the IFS $\mathcal{I}$ is uniformly contractive on $U$, therefore it defines a unique attractor $K \subset U$, i.e. a non-empty compact set such that

$$
K=\bigcup_{i=1}^{m} f_{i}(K) .
$$

Such a $K$ is called a self-conformal set. The set $K$ has a natural symbolic representation: let $\Lambda=\{1, \ldots, m\}$ be the alphabet and let $\Lambda^{\mathbb{N}}$ be the symbolic space with $m$ letters. For each $i=i_{1} \cdots i_{n} \in \Lambda^{n}$ denote by

$$
f_{i}=f_{i_{1}} \circ \cdots \circ f_{i_{n}}
$$

and for $\underline{i}=i_{1} i_{2} \cdots \in \Lambda^{\mathbb{N}}$ and $n \geq 1$ denote by $\left.\underline{i}\right|_{n}=i_{1} \cdots i_{n}$. Fix a point $x_{0} \in U$. Then we may define a map $\Phi: \Lambda^{\mathbb{N}} \rightarrow U$ by

$$
\Phi(\underline{i})=\lim _{n \rightarrow \infty} f_{\left.\underline{i}\right|_{n}}\left(x_{0}\right) .
$$

Since all $f_{i}$ are injective, the above limit always exists and it does not depend on the choice of $x_{0}$. Its image is the self-conformal set $K$ and $\Phi$ is called the canonical map.

For $i=i_{1} \cdots i_{n} \in \Lambda^{n}$ denote by $[i]=\left\{\underline{i} \in \Lambda^{\mathbb{N}}:\left.\underline{i}\right|_{n}=i\right\}$ the cylinder in $\Lambda^{\mathbb{N}}$ encoded by $i$. Let $\mathcal{B}$ denote the $\sigma$-algebra generated by cylinders. Let $\sigma$ denote the left-shift operator on $\Lambda^{\mathbb{N}}$. Let $\varphi: \Lambda^{\mathbb{N}} \rightarrow \mathbb{R}$ be a Hölder potential on $\Lambda^{\mathbb{N}}$ and let $\mu_{\varphi}$ denote its Gibbs measure (see Section 2.4 for precise definition). We are interested in the orthogonal projections of the push-forward measure

$$
\Phi \mu_{\varphi}=\mu_{\varphi} \circ \Phi^{-1}
$$

on the self-conformal set $K$. Before stating our main result, we shall present an analogue of the dense rotations condition in the self-conformal case. Let $G=S O(d, \mathbb{R})$ and define a map $\phi: \Lambda^{\mathbb{N}} \rightarrow G$ as follows:

$$
\phi(\underline{i})=O_{i_{1}}(\Phi(\sigma \underline{i})) \text { for } \underline{i}=i_{1} i_{2} \cdots .
$$

Then we may define the skew product $\sigma_{\phi}: \Lambda^{\mathbb{N}} \times G \rightarrow \Lambda^{\mathbb{N}} \times G$ as

$$
\sigma_{\phi}(\underline{i}, O)=(\sigma \underline{i}, O \phi(\underline{i})) .
$$

We assume

(A2) $\sigma_{\phi}$ has a dense orbit in $\Lambda^{\mathbb{N}} \times G$. 
By a compact group extension theorem (see Subsection 2.5), this implies that the dynamical system

$$
\left(\Lambda^{\mathbb{N}} \times G, \mathcal{B} \otimes \mathcal{B}_{G}, \sigma_{\phi}, \mu_{\varphi} \times \xi\right)
$$

is ergodic, where $\xi$ is the normalised right-invariant Haar measure on $G$, and $\mathcal{B}_{G}$ is its Borel $\sigma$-algebra.

Remark 1.2. In the above dynamical system, (A2) is equivalent to topological transitivity, that is, for any non-empty open sets $U, V \in \Lambda^{\mathbb{N}} \times G$, there exists $n>1$ such that $\sigma_{\phi}^{n}(U) \cap V$ is non-empty. See 23] for details. We shall prove (see Lemma 2.2), that if there exists a dense orbit $\left\{O_{\left.\underline{i}\right|_{n}}\left(\Phi\left(\sigma^{n} \underline{i}\right)\right)\right\}_{n \geq 1}$ in $G$, then this implies topological transitivity.

Now we are ready to state our main result:

Theorem 1.3. Under assumptions (A0), (A1) and (A2), for all $\pi \in \Pi_{d, k}$ we have

$$
\operatorname{dim}_{H} \pi \Phi \mu_{\varphi}=\min \left\{k, \operatorname{dim}_{H} \Phi \mu_{\varphi}\right\} .
$$

With the same approach as in [12] we can also prove the following.

Corollary 1.4. Under assumptions (A0), (A1) and (A2), for all $C^{1}$-maps $h: K \rightarrow \mathbb{R}^{k}$ without singular points,

$$
\operatorname{dim}_{H} h \Phi \mu_{\varphi}=\min \left\{k, \operatorname{dim}_{H} \Phi \mu_{\varphi}\right\} .
$$

It is well-known that if the self-conformal set $K$ satisfies the open set condition (OSC) then there exists a Gibbs measure $\mu$ of a Hölder potential on $\Lambda^{\mathbb{N}}$ such that $\operatorname{dim}_{H} \Phi \mu=$ $\operatorname{dim}_{H} K$, and $\mu$ is equivalent to $\operatorname{dim}_{H} K$-dimensional Hausdorff measure (see [4, 19] for example). This implies the following.

Corollary 1.5. Under assumptions (AO), (A1) and (A2), as well as the OSC, for all $C^{1}$-maps $h: K \rightarrow \mathbb{R}^{k}$ without singular points,

$$
\operatorname{dim}_{H} h(K)=\min \left\{k, \operatorname{dim}_{H} K\right\} .
$$

Remark 1.6. The $O S C$ in the above Corollary can be relaxed to the so-called strong variational principle: there exists a Hölder potential $\varphi$ such that the corresponding Gibbs measure $\mu_{\varphi}$ satisfies $\operatorname{dim}_{H} \mu_{\varphi}=\operatorname{dim}_{H} K$. We believe that this should hold for our family of self-conformal sets.

By applying Corollary 1.5 to $C^{1}$-maps $h(x)=|x-a|$ outside a neighborhood of $a \in K$ we deduce that Falconer's distance set conjecture (see [22] and the references therein for most recent developments), is true for this family of self-conformal sets:

Corollary 1.7. Under assumptions (AO), (A1) and (A2), as well as the OSC, if $\operatorname{dim}_{H} K \geq$ 1 , then for $a \in K$,

$$
\operatorname{dim}_{H}\{|x-a|: x \in K\}=\operatorname{dim}_{H}\{|x-y|: x, y \in K\}=1 .
$$

Remark 1.8. Since self-conformal sets with OSC are Ahlfors-David regular, when $d=2$ and $\operatorname{dim}_{H} K>1$, Corollary 1.7 is actually a direct consequence of Theorem 1.1 in [22]. But the case when $d>2$ or $\operatorname{dim}_{H} K=1$ is new to the best of our knowledge.

Now we give an example of self-conformal sets which satisfy all of our assumptions. According to Theorem 14.15 in [5], when $|c|>\frac{1}{4}(5+2 \sqrt{6})=2.475 \ldots$, the Julia set $J_{f_{c}}$ defined by the quadratic polynomial $f_{c}(z)=z^{2}+c$ is totally disconnected, and is the attractor of the conformal IFS

$$
\left\{f_{1}(z)=\sqrt{z-c}, \quad f_{2}(z)=-\sqrt{z-c}\right\} .
$$


We may take $U=\left\{z:|z|<|2 c|^{1 / 2}\right\}$. It is easy to see that $U$ is bounded, open and convex, and for all $z \in U$,

$$
\left|f_{i}^{\prime}(z)\right|=\frac{1}{2}|z-c|^{-1 / 2} \leq \frac{1}{2}\left(|c|-|2 c|^{1 / 2}\right)^{-1 / 2}<1 .
$$

This verifies assumptions (A0) and (A1), as well as the OSC. Now take a fixed point $\alpha=\frac{1+\sqrt{1-4 c}}{2} \in J_{f_{c}}$ so that $f_{1}(\alpha)=\alpha$. We have

$$
f_{1}^{\prime}(\alpha)=\frac{1}{2 \sqrt{\alpha-c}}=\frac{1}{2 \alpha}=\frac{1}{1+\sqrt{1-4 c}} .
$$

Therefore, if

$$
\frac{\arg f^{\prime}(\alpha)}{\pi}=\frac{\arg (1+\sqrt{1-4 c})}{\pi} \text { is irrational, }
$$

then $\left\{O_{\left.\underline{i}\right|_{n}}\left(x_{\sigma^{n} \underline{i}}\right)\right\}_{n \geq 1}$ is dense in $S O(2, \mathbb{R})$ for $\underline{i}=111 \cdots$. This verifies assumption (A2).

Remark 1.9. The above example is a particular case of hyperbolic Julia sets. It is worth mentioning that in 2 Bedford, Fisher and Urbański showed that the scenery flow of hyperbolic Julia sets (a geometric realization of our dynamical system $\left(\Lambda^{\mathbb{N}} \times G, \mathcal{B} \otimes \mathcal{B}_{G}, \mu \times \xi, \sigma_{\phi}\right)$ ) is ergodic in all cases with the exception of the following:

i) the Julia set $J_{f}$ is a geometric circle and $f$ is biholomorphically conjugate to a finite Blaschke product, or

ii) the Julia set $J_{f}$ is totally disconnected and $J_{f}$ is contained in a real-analytic curve with self-intersections (if any) lying outside the Julia set.

It would be interesting to see if for hyperbolic Julia sets, our assumption (A2) can be replaced by the above criteria.

The rest of the paper is organised as follows. In Section 2 we will first go through some background on symbolic space and self-conformal sets. We present the bounded distortion property which is satisfied in our setting. We will briefly mention the thermodynamic formalism which defines the Gibbs measure, through which we obtain an ergodic dynamical system. From this ergodicity we know from [9] that our Gibbs measure $\Phi \mu_{\varphi}$ is exact dimensional (see Section 2.6 for the definition). A theorem on compact group extension will show us that the skew product of this dynamical system with $G$ is also ergodic. From here, having stated some definitions of entropy and dimension, we move on to the dimension of the projections of the self-conformal Gibbs measures. Section 3 uses the methods of [7, 12], to prove that the dimension of the projections of these measures takes the 'expected' value for $\xi$-almost all $\pi \in \Pi_{d, k}$. Then following a similar argument of Hochman and Shmerkin [12] we may extend the value to all $\pi \in \Pi_{d, k}$ and to all $C^{1}$-maps without singular points.

\section{Preliminaries}

2.1. Symbolic space. Let $\Lambda=\{1, \ldots, m\}$ be the alphabet on $m \geq 2$ symbols. Let $\Lambda^{*}=\bigcup_{n \geq 1} \Lambda^{n}$ be the set of finite words. For $i \in \Lambda^{*}$ let $|i|$ denote the length of the word. Let $\Lambda^{\mathbb{N}}$ be the symbolic space of infinite sequences from the alphabet. For $\underline{i} \in \Lambda^{\mathbb{N}}, n \geq 1$, let $\left.\underline{i}\right|_{n} \in \Lambda^{n}$ be the first $n$ digits of $\underline{i}$. For $i \in \Lambda^{n}$, let $[i]=\left\{\underline{i} \in \Lambda^{\mathbb{N}}:\left.\underline{i}\right|_{n}=i\right\}$ be the cylinder rooted at $i$. We may endow $\Lambda^{\mathbb{N}}$ with the standard metric $d_{\rho}$ with respect to a real number $\rho \in(0,1)$, that is, for $\underline{i}, \underline{j} \in \Lambda^{\mathbb{N}}$,

$$
d_{\rho}(\underline{i}, \underline{j})=\rho^{\inf \left\{n \geq 0:\left.\underline{i}\right|_{n} \neq\left.\underline{j}\right|_{n}\right\}},
$$


with the convention that $\left.\underline{i}\right|_{0}=\emptyset$ for all $\underline{i} \in \Lambda^{\mathbb{N}}$. Then $\left(\Lambda^{\mathbb{N}}, d_{\rho}\right)$ is a compact metric space. Let $\mathcal{B}$ be its Borel $\sigma$-algebra. Define the left shift map $\sigma$ by $\sigma(\underline{i})=\left(i_{n+1}\right)_{n \geq 1}$ for $\underline{i}=\left(i_{n}\right)_{n \geq 1} \in \Lambda^{\mathbb{N}}$.

2.2. Self-conformal sets. Let $\mathcal{I}$ be an iterated function system (IFS) as in (1.1) of conformal maps defined on a bounded open connected subset $U \subseteq \mathbb{R}^{d}$ with non-empty compact attractor $K \subseteq \mathbb{R}^{d}$ satisfying (1.2). Since $\mathcal{I}$ is uniformly contractive on $U$, one can find a connected open set $V$ such that $K \subset V \subset \bar{V} \subset U$ and $\min \{\operatorname{dist}(K, \partial V), \operatorname{dist}(V, \partial U)\}>$ 0 . Recall that $\Phi: \Lambda^{\mathbb{N}} \rightarrow K$ is the canonical projection, that is, $\Phi(\underline{i})=\lim _{n \rightarrow \infty} f_{\left.\underline{i}\right|_{n}}\left(x_{0}\right)$ for some $x_{0} \in U$. We shall also use the notation $x_{\underline{i}}=\Phi(\underline{i})$ for $\underline{i} \in \Lambda^{\mathbb{N}}$.

2.3. Bounded distortion. For $x \in V$ and $i=i_{1} \cdots i_{n} \in \Lambda^{n}$ we may write

$$
f_{i}^{\prime}(x)=r_{i}(x) O_{i}(x)
$$

where

$$
\begin{aligned}
r_{i}(x) & =r_{i_{1}}\left(f_{i_{2}} \circ \cdots \circ f_{i_{n}}(x)\right) \cdot r_{i_{2}}\left(f_{i_{3}} \circ \cdots \circ f_{i_{n}}(x)\right) \cdots r_{i_{n}}(x), \\
O_{i}(x) & =O_{i_{1}}\left(f_{i_{2}} \circ \cdots \circ f_{i_{n}}(x)\right) \cdot O_{i_{2}}\left(f_{i_{3}} \circ \cdots \circ f_{i_{n}}(x)\right) \cdots O_{i_{n}}(x) .
\end{aligned}
$$

It is well-known that in a simply-connected complex domain every holomorphic function is analytic. Therefore we have the following bounded distortion property: there exists a constant $C_{1}>0$ such that for all $i \in \Lambda^{*}$ and $x, y \in V$,

$$
\frac{r_{i}(x)}{r_{i}(y)} \leq C_{1}
$$

To see this, for $i=i_{1} \cdots i_{n} \in \Lambda^{n}$ we have

$$
\log \frac{r_{i}(x)}{r_{i}(y)}=\sum_{k=1}^{n} \log r_{i_{k}}\left(f_{i_{k+1} \cdots i_{n}}(x)\right)-\log r_{i_{k}}\left(f_{i_{k+1} \cdots i_{n}}(y)\right) .
$$

By the smoothness of $\log r_{i}$ one can find a constant $\widetilde{C}_{1}$ such that for all $x, y \in V$ and $i \in \Lambda$,

$$
\left|\log r_{i}(x)-\log r_{i}(y)\right| \leq \widetilde{C}_{1}|x-y| .
$$

On the other hand, by (A1), one can find another constant $\widetilde{C}_{1}^{\prime}$ such that for all $x, y \in V$, $n \geq 1$ and $i=i_{1} \cdots i_{n} \in \Lambda^{n}$,

$$
\left|f_{i}(x)-f_{i}(y)\right| \leq \widetilde{C}_{1}^{\prime}\left(r^{*}\right)^{n} .
$$

Combining these two inequalities we get from (2.3) that

$$
\log \frac{r_{i}(x)}{r_{i}(y)} \leq \widetilde{C}_{1} \widetilde{C}_{1}^{\prime} \sum_{k=1}^{n}\left(r^{*}\right)^{n-k} \leq \widetilde{C}_{1} \widetilde{C}_{1}^{\prime} \frac{1}{1-r^{*}}:=\log C_{1} .
$$

The bounded distortion also implies the following fact: there exists a constant $C_{2}>0$ such that for all $i \in \Lambda^{*}$ and all $x, y \in V$,

$$
C_{2}^{-1} \bar{r}_{i}|x-y| \leq\left|f_{i}(x)-f_{i}(y)\right| \leq C_{2} \bar{r}_{i}|x-y|,
$$

where for $i \in \Lambda^{*}$ we denote by $\bar{r}_{i}=\sup \left\{r_{i}(x): x \in V\right\}$. For a proof see [18, Lemma 2.2] for example (note that the proof does not require any separation condition). 
2.4. Gibbs measures. Let $\varphi$ be a Hölder potential defined on $\Lambda^{\mathbb{N}}$. This means there exist constants $\kappa>0$ and $\beta \in(0,1)$ such that for $n \geq 1$,

$$
\operatorname{Var}_{n}(\varphi):=\sup _{i \in \Lambda^{n}} \sup _{\underline{i}, \underline{j} \in[i]}|\varphi(\underline{i})-\varphi(\underline{j})| \leq \kappa \beta^{n} .
$$

For $n \geq 1$ the $n$ th-order Birkhoff sum of $\varphi$ over $\sigma$ is defined as

$$
S_{n} \varphi(\underline{i})=\sum_{k=0}^{n-1} \varphi \circ \sigma^{k}(\underline{i}),
$$

for $\underline{i} \in \Lambda^{\mathbb{N}}$. The topological pressure of $\varphi$ on $\Lambda^{\mathbb{N}}$ is given by

$$
P(\varphi)=\lim _{n \rightarrow \infty} \frac{1}{n} \log \sum_{j \in \Lambda^{n}} \exp \left(\max _{\underline{i} \in[j]} S_{n} \varphi(\underline{i})\right),
$$

where the existence of the limit can be proved using the sub-additive property of the logarithm on the right hand side. It follows from the thermodynamic formalism developed by Sinai, Ruelle, Bowen and Walters [3, 20] that there exists a unique ergodic measure $\mu_{\varphi}$, namely the Gibbs measure of $\varphi$ on $\left(\Lambda^{\mathbb{N}}, \sigma\right)$, such that for any $\underline{i} \in \Lambda^{\mathbb{N}}, n \geq 0$ and $\underline{j} \in\left[\left.\underline{i}\right|_{n}\right]$,

$$
e^{-\operatorname{Var}_{n}(\varphi)} \leq \frac{\mu_{\varphi}\left(\left[\left.\underline{i}\right|_{n}\right]\right)}{\exp \left(S_{n} \varphi(\underline{j})-n P(\varphi)\right)} \leq e^{\operatorname{Var}_{n}(\varphi)} .
$$

Also, $\mu_{\varphi}$ possesses the quasi-Bernoulli property:

$$
e^{-\kappa \frac{\beta^{|i|}}{1-\beta}-\kappa \beta^{|j|}} \mu_{\varphi}([i]) \mu_{\varphi}([j]) \leq \mu_{\varphi}([i j]) \leq e^{\kappa \frac{\beta^{|i|}}{1-\beta}+\kappa \beta^{|j|}} \mu_{\varphi}([i]) \mu_{\varphi}([j])
$$

for all $i, j \in \Lambda^{*}$. (Here we have been more precise on the quasi-Bernoulli constant $e^{\kappa \frac{\beta^{|i|}}{1-\beta}+\kappa \beta^{|j|}}$ in terms of the length of $i$ and $j$.) In particular, when the potential function $\varphi: \Lambda^{\mathbb{N}} \rightarrow \mathbb{R}$ takes the values $\varphi(\underline{i})=\log p_{\left.\underline{i}\right|_{1}}$ for a fixed vector $p=\left(p_{i}\right)_{i \in \Lambda}$, such that $0<p_{i}<1$ and $\sum_{i \in \Lambda} p_{i}=1$, noting that it is Lipschitz on $\left(\Lambda^{\mathbb{N}}, d_{\rho}\right)$, then we can define a Gibbs measure $\mu_{p}$ on $\Lambda^{\mathbb{N}}$ by:

$$
\begin{aligned}
\mu_{p}([i]) & =\exp S_{n} \varphi(\underline{i}) \\
& =p_{i_{1}} \cdots p_{i_{n}},
\end{aligned}
$$

for $\underline{i} \in[i]$. This is simply the Bernoulli measure on $\Lambda^{\mathbb{N}}$.

2.5. The compact group extension. We will now deal with the system $\left(\Lambda^{\mathbb{N}}, \mathcal{B}, \sigma, \mu\right)$ and its compact group extensions, where $\mu=\mu_{\varphi}$ is a Gibbs measure with respect to a Hölder potential $\varphi$. Recall that $G=S O(d, \mathbb{R})$ is a compact Lie group with Borel $\sigma$-algebra $\mathcal{B}_{G}$ and we have defined the map $\phi: \Lambda^{\mathbb{N}} \rightarrow G$ as

$$
\phi(\underline{i})=O_{i_{1}}(\Phi(\sigma \underline{i})) \text { for } \underline{i}=i_{1} i_{2} \cdots .
$$

By the smoothness of conformal maps it is easy to see that $\phi$ is Hölder on $\left(\Lambda^{\mathbb{N}}, d_{\rho}\right)$. We may define the skew product $\sigma_{\phi}: \Lambda^{\mathbb{N}} \times G \rightarrow \Lambda^{\mathbb{N}} \times G$ as follows:

$$
\sigma_{\phi}(\underline{i}, O)=(\sigma \underline{i}, O \phi(\underline{i})) \text {. }
$$

It is easy to verify that the product measure $\mu \times \xi$ is $\sigma_{\phi}$-invariant, where $\xi$ is the rightinvariant normalised Haar measure on $G$. Under (A2) we have that $\sigma_{\phi}$ has a dense orbit in $\Lambda^{\mathbb{N}} \times G$.

Proposition 2.1. The dynamical system $\left(\Lambda^{\mathbb{N}} \times G, \mathcal{B} \otimes \mathcal{B}_{G}, \mu \times \xi, \sigma_{\phi}\right)$ is ergodic.

Proof. This directly follows from [17, Corollary 4.5]. 
Here we give a sufficient assumption (equivalent to the dense rotations condition when the conformal functions are similarities) to achieve topological transitivity, and therefore (A2).

Lemma 2.2. Assume that for some $\underline{i} \in \Lambda^{\mathbb{N}}$,

$$
\left\{O_{\left.\underline{i}\right|_{n}}\left(x_{\sigma^{n} \underline{i}}\right)\right\}_{n \geq 1} \text { is dense in } G \text {. }
$$

Then the skew product $\sigma_{\phi}$ is topologically transitive.

Proof. Recall the notation $x_{\underline{i}}=\Phi(\underline{i})$ for $\underline{i} \in \Lambda^{\mathbb{N}}$. Fix $\underline{i} \in \Lambda^{\mathbb{N}}$ so that $\left\{O_{\left.\underline{i}\right|_{n}}\left(x_{\sigma^{n}} \underline{i}\right)\right\}_{n \geq 1}$ is dense in $G$. Recall the definition of the skew product $\sigma_{\phi}$,

$$
\sigma_{\phi}: \Lambda^{\mathbb{N}} \times G \rightarrow \Lambda^{\mathbb{N}} \times G, \quad(\underline{i}, O) \rightarrow\left(\sigma \underline{i}, O \cdot O_{\left.\underline{i}\right|_{1}}\left(x_{\sigma \underline{i}}\right)\right) .
$$

Take $U, V$ open sets in $\Lambda^{\mathbb{N}} \times G$. Then there exist finite words $u, v$ such that $[u] \subset \pi_{\Lambda^{\mathbb{N}}}(U)$, and $[v] \subset \pi_{\Lambda^{\mathbb{N}}}(V)$, where $\pi_{X}$ denotes the projection onto $X$. For $O \in \pi_{G}(V)$, there exists $n \geq 1$ such that

$$
\begin{aligned}
O \cdot O_{u \underline{\left.\underline{i}\right|_{|u|+n}}}\left(x_{\sigma^{|u|+n}(u \underline{i})}\right) & =O \cdot O_{u}\left(x_{i_{1} i_{2} \ldots}\right) \cdot O_{i_{1} \cdots i_{n}}\left(x_{i_{n+1} i_{n+2} \cdots}\right) \\
& =O \cdot O_{u}\left(x_{\underline{i}}\right) \cdot O_{\left.\underline{i}\right|_{n}}\left(x_{\sigma^{n} \underline{i}}\right) \in \pi_{G}(U) .
\end{aligned}
$$

This follows from the fact that $O, O_{u}\left(x_{\underline{i}}\right)$ are fixed and the orbit of $O_{\underline{i} \mid n}\left(x_{\sigma^{n} \underline{\underline{n}}}\right)$ is dense. Now consider an infinite word

$$
\underline{k}=\left.u \underline{i}\right|_{n} v \ldots,
$$

where the symbols following $v$ are arbitrary. Then $\left(\underline{k}, O \cdot O_{\left.u \underline{i}\right|_{|u|+n}}\left(x_{\sigma^{|u|+n}(u \underline{i})}\right)\right) \in U$, and

$$
\sigma_{\phi}^{|u|+n}\left(\underline{k}, O \cdot O_{\left.u \underline{i}\right|_{|u|+n}}\left(x_{\sigma^{|u|+n}(u \underline{i})}\right)\right)=(v \ldots, O) \in V .
$$

In particular, if there exists a finite word $u$ such that $O_{u}\left(x_{\bar{u}}\right)$ is an irrational rotation, where $\bar{u}=u и u \cdots$ denotes the periodic infinite word of $u$, then (2.8) is true.

2.6. Dimension and entropy. Let $g: Y \rightarrow Z$ be a continuous mapping between two metric spaces $Y$ and $Z$. For a Borel measure $\nu$ on $Y$, write

$$
g \nu=\nu \circ g^{-1},
$$

for the pull-back measure of $\nu$ on $Z$ through $\varphi$. For a measure $\nu$ and $x \in \operatorname{supp}(\nu)$, let

$$
D_{\nu}(x)=\lim _{r \rightarrow 0} \frac{\log \nu(B(x, r))}{\log r},
$$

whenever the limit exists, where $B(x, r)$ is the closed ball of centre $x$ and radius $r$. If for some $\alpha \geq 0$, we have $D_{\nu}(x)=\alpha$ for $\nu$-a.e. $x$, we say that $\nu$ is exact dimensional.

For $0<r<1$ and $\nu$, a probability measure supported by a compact subset $A$ of $\mathbb{R}^{2}$, let

$$
H_{r}(\nu)=-\int_{A} \log \nu(B(x, r)) \nu(d x)
$$

be the $r$-scaling entropy of $\nu$. Note that, writing $\mathcal{M}$ for the probability measures supported by $A$, the map $H_{r}: \mathcal{M} \rightarrow \mathbb{R} \cup\{\infty\}$ need not be continuous in the weak- $\star$ topology. However, $H_{r}$ is lower semicontinuous as it may be expressed as the limit of an increasing sequence of continuous functions of the form $\nu \rightarrow \int \max \left\{k, \log \left(1 / \int f_{k}(x-y) \nu(d y) \nu(d x)\right)\right\}$, where $f_{k}$ is a decreasing sequence of continuous functions approximating $\chi_{B(0, r)}$. The lower entropy dimension of $\nu$ is defined as

$$
\operatorname{dim}_{e} \nu=\liminf _{r \rightarrow 0} \frac{H_{r}(\nu)}{-\log r}
$$


and the Hausdorff dimension of $\nu$ is $\operatorname{dim}_{H} \nu=\inf \left\{\operatorname{dim}_{H} A: \nu(A)>0\right\}$. Then

$$
\operatorname{dim}_{H} \nu \leq \operatorname{dim}_{e} \nu
$$

with equality when $\nu$ is exact dimensional, for details see [8]. From [9] we have that the self-conformal measure $\Phi \mu$ is exact dimensional.

\section{Dimension of PROJECTIONS}

Let $B=B(0, R)$ be the closed ball of radius $R$, where $R=\max \{|x|: x \in V\}$. Denote by $\mathcal{M}$ the family of probability measures on $B$ and let $\mathcal{B}_{\star}$ be its weak- $\star$ topology. Denote by $C(\mathcal{M})$ the family of all continuous functions on $\mathcal{M}$. We use the separability of $C(\mathcal{M})$ in $\|\cdot\|_{\infty}$ to obtain convergence of ergodic averages for all $h \in C(\mathcal{M})$.

Proposition 3.1. We have that for $\xi$-a.e. $O \in G$ and $\mu$-a.e. $\underline{i} \in \Lambda^{\mathbb{N}}$,

$$
\lim _{N \rightarrow \infty} \frac{1}{N} \sum_{n=0}^{N-1} h\left(O \cdot O_{\left.\underline{\underline{i}}\right|_{n}}\left(\Phi\left(\sigma^{n} \underline{i}\right)\right) \Phi \mu\right)=\mathbb{E}_{\mu \times \xi}(h(O \Phi \mu))
$$

for all $h \in C(\mathcal{M})$.

Proof. Let $\left\{h_{k}\right\}_{k \geq 1}$ be a countable dense sequence in $C(\mathcal{M})$. If we write

$$
M: \Lambda^{\mathbb{N}} \times G \ni(\underline{i}, O) \rightarrow O \Phi \mu \in \mathcal{M},
$$

then it is easy to verify that for $n \geq 0$,

$$
M \circ \sigma_{\phi}^{n}(\underline{i}, O)=O \cdot O_{\left.\underline{i}\right|_{n}}\left(\Phi\left(\sigma^{n} \underline{i}\right)\right) \Phi \mu .
$$

Since we know that $\left(\Lambda^{\mathbb{N}} \times G, \mathcal{B} \otimes \mathcal{B}_{G}, \mu \times \xi, \sigma_{\phi}\right)$ is ergodic, we have that for $\xi$-a.e. $O$ and $\mu$-a.e. $\underline{i}$,

$$
\lim _{N \rightarrow \infty} \frac{1}{N} \sum_{n=1}^{N} h_{k}\left(O \cdot O_{\left.\underline{i}\right|_{n}}\left(\Phi\left(\sigma^{n} \underline{i}\right)\right) \Phi \mu\right)=\mathbb{E}_{\mu \times \xi}\left(h_{k}(O \Phi \mu)\right),
$$

for all $k \geq 1$. For any $h \in C(\mathcal{M})$, take a subsequence $\left\{h_{k}^{\prime}\right\}_{k \geq 1}$ of $\left\{h_{k}\right\}_{k \geq 1}$ that converges to $h$. On the one hand, since $\mathcal{M}$ is compact, $h$ is bounded, so by the uniform convergence in $\|\cdot\|_{\infty}$,

$$
\lim _{k \rightarrow \infty} \mathbb{E}_{\mu \times \xi}\left(h_{k}^{\prime}(O \Phi \mu)\right)=\mathbb{E}_{\mu \times \xi}(h(O \Phi \mu)) .
$$

On the other hand, for each $N$,

$$
\left|\frac{1}{N} \sum_{n=0}^{N-1} h_{k}^{\prime}\left(O \cdot O_{\left.\underline{i}\right|_{n}}\left(\Phi\left(\sigma^{n} \underline{i}\right)\right) \Phi \mu\right)-\frac{1}{N} \sum_{n=0}^{N-1} h\left(O \cdot O_{\left.\underline{i}\right|_{n}}\left(\Phi\left(\sigma^{n} \underline{i}\right)\right) \Phi \mu\right)\right| \leq\left\|h_{k}^{\prime}-h\right\|_{\infty} .
$$

Thus the limit

$$
\lim _{N \rightarrow \infty} \frac{1}{N} \sum_{n=0}^{N-1} h\left(O \cdot O_{\left.\underline{i}\right|_{n}}\left(\Phi\left(\sigma^{n} \underline{i}\right)\right) \Phi \mu\right)
$$

exists and equals $\lim _{k \rightarrow \infty} \mathbb{E}_{\mu \times \xi}\left(h_{k}^{\prime}(O \Phi \mu)\right)=\mathbb{E}_{\mu \times \xi}(h(O \Phi \mu))$, for $\xi$-a.e. $O \in G$ and $\mu$-a.e. $\underline{i} \in \Lambda^{\mathbb{N}}$. 
3.1. Lower bound for the dimension of projections. First we shall prove the following lemma regarding distortions of conformal maps under projections.

Lemma 3.2. There exists a constant $C_{3}$ such that for all $\pi \in \Pi_{d, k}, n \geq 1, i \in \Lambda^{n}$ and $x, y, z \in V$ one has

$$
\begin{array}{r}
C_{1}^{-1} \bar{r}_{i}\left|\pi O_{i}(z)(x-y)\right|-C_{3} \bar{r}_{i}(|z-x|+|z-y|)|x-y| \leq\left|\pi f_{i}(x)-\pi f_{i}(y)\right| \\
\leq \bar{r}_{i}\left|\pi O_{i}(z)(x-y)\right|+C_{3} \bar{r}_{i}(|z-x|+|z-y|)|x-y|,
\end{array}
$$

where $C_{1}$ is as in (2.2).

Proof. For $\pi \in \Pi_{d, k}$ one can find a rotation $O_{\pi} \in S O(d, \mathbb{R})$ such that

$$
O_{\pi} \pi(z)=\left(P_{j}\left(O_{\pi} z\right)\right)_{1 \leq j \leq k}
$$

for all $z \in \mathbb{R}^{d}$, where $P_{j}: \mathbb{R}^{d} \rightarrow \mathbb{R}$ is the coordinate function $P_{j}\left(x_{1}, \ldots, x_{d}\right)=x_{j}$. We will view a function $f: \mathbb{R}^{d} \rightarrow \mathbb{R}^{d}$ as $f\left(x_{1}, \ldots, x_{d}\right)=\left(f^{1}(x), \ldots, f^{d}(x)\right)$. We fix $1 \leq j \leq k$ and consider $f^{j}: \mathbb{R}^{d} \rightarrow \mathbb{R}$. For $i \in \Lambda^{*}$ and $x, y \in V$ we have

$$
P_{j}\left(O_{\pi} \pi\left(f_{i}(x)-f_{i}(y)\right)\right)=P_{j}\left(O_{\pi} f_{i}(x)-O_{\pi} f_{i}(y)\right) .
$$

For $t \in[0,1]$ define

$$
g(t)=P_{j}\left(t\left(O_{\pi} f_{i}(x)-O_{\pi} f_{i}(y)\right)-O_{\pi} f_{i}(y+t(x-y))\right) .
$$

Since $g(0)=g(1)=-P_{j}\left(O_{\pi} f_{i}(y)\right)$, by Rolle's theorem there exists $t \in(0,1)$ such that $g^{\prime}(t)=0$, which means that

$$
P_{j}\left(O_{\pi} f_{i}(x)-O_{\pi} f_{i}(y)\right)=P_{j}\left(O_{\pi} f_{i}^{\prime}\left(z_{x, y}^{j}\right)(x-y)\right),
$$

where $z_{x, y}^{j}=y+t(x-y)$ is some point lying in the line segment between $x$ and $y$. Writing $f_{i}^{\prime}\left(z_{x, y}^{j}\right)=r_{i}\left(z_{x, y}^{j}\right) O_{i}\left(z_{x, y}^{j}\right)$, for $z \in V$ we have

$$
\begin{aligned}
& P_{j}\left(O_{\pi} \pi\left(f_{i}(x)-f_{i}(y)\right)\right) \\
= & P_{j}\left(O_{\pi} f_{i}(x)-O_{\pi} f_{i}(y)\right) \\
= & r_{i}\left(z_{x, y}^{j}\right) P_{j}\left(O_{\pi} O_{i}\left(z_{x, y}^{j}\right)(x-y)\right) \\
= & r_{i}\left(z_{x, y}^{j}\right) P_{j}\left(O_{\pi} O_{i}(z)(x-y)\right)+r_{i}\left(z_{x, y}^{j}\right) P_{j}\left(O_{\pi}\left(O_{i}\left(z_{x, y}^{j}\right)-O_{i}(z)\right)(x-y)\right) \\
= & r_{i}\left(z_{x, y}^{j}\right) P_{j}\left(\pi\left(O_{i}(z)(x-y)\right)\right)+r_{i}\left(z_{x, y}^{j}\right) P_{j}\left(\pi\left(\left(O_{i}\left(z_{x, y}^{j}\right)-O_{i}(z)\right)(x-y)\right)\right) .
\end{aligned}
$$

This holds for all $1 \leq j \leq k$, and so

$$
\begin{aligned}
& \left(O_{\pi} \pi\left(f_{i}(x)-f_{i}(y)\right)\right) \\
& =\left(r_{i}\left(z_{x, y}^{1}\right) P_{1}\left(\pi\left(O_{i}(z)(x-y)\right)\right)+r_{i}\left(z_{x, y}^{1}\right) P_{1}\left(\pi\left(\left(O_{i}\left(z_{x, y}^{1}\right)-O_{i}(z)\right)(x-y)\right)\right),\right. \\
& \left.\quad \ldots, r_{i}\left(z_{x, y}^{k}\right) P_{k}\left(\pi\left(O_{i}(z)(x-y)\right)\right)+r_{i}\left(z_{x, y}^{k}\right) P_{k}\left(\pi\left(\left(O_{i}\left(z_{x, y}^{k}\right)-O_{i}(z)\right)(x-y)\right)\right)\right) .
\end{aligned}
$$

By the smoothness of $O_{i}$ and (2.2), as well as the fact that $O_{\pi}$ is isometric, one can find a constant $\widetilde{C}_{3}$ such that

$$
\left|O_{\pi} \pi\left(f_{i}(x)-f_{i}(y)\right)\right| \leq \bar{r}_{i}\left|\pi\left(O_{i}(z)(x-y)\right)\right|+\widetilde{C}_{3} \bar{r}_{i} \max _{1 \leq j \leq k}\left|z_{x, y}^{j}-z\right||x-y|
$$

as well as

$$
C_{1}^{-1} \bar{r}_{i}\left|\pi O_{i}(z)(x-y)\right| \leq\left|\pi f_{i}(x)-\pi f_{i}(y)\right|+\widetilde{C}_{3} \bar{r}_{i} \max _{1 \leq j \leq k}\left|z_{x, y}^{j}-z\right||x-y|
$$

Finally note that $z_{x, y}$ lies in the line segment between $x$ and $y$, therefore

$$
\max _{1 \leq j \leq k}\left|z_{x, y}^{j}-z\right| \leq|z-x|+|z-y| \text {. }
$$


Let $\rho=\max \left\{r_{i}(x): i \in \Lambda, x \in K\right\}$. By (A1) we have $\rho<1$. Recall that for $i \in \Lambda^{*}$ we denote by $\bar{r}_{i}=\sup \left\{r_{i}(x): x \in V\right\}$. For each $q \geq 1$ we redefine the alphabet used for symbolic space to obtain one in which the contraction ratios do not vary too much:

$$
\Lambda_{q}=\left\{i \in \Lambda^{*}: \bar{r}_{i} \leq \rho^{q}<\bar{r}_{i^{-}}\right\},
$$

where $i^{-}=i_{1} i_{2} \cdots i_{n-1}$. Define $\underline{r}=\inf \left\{r_{i}(x): i \in \Lambda, x \in V\right\}$. Since $f_{i}$ are conformal and $\bar{V} \subset U$ is compact, we have $\underline{r}>0$. By definition, for $i \in \Lambda_{q}$ one has

$$
\underline{r} \rho^{q}<\bar{r}_{i} \leq \rho^{|i|} \text { and } \underline{r}^{|i|} \leq \bar{r}_{i} \leq \rho^{q} .
$$

This implies that for $i \in \Lambda_{q}$,

$$
q \frac{\log \rho}{\log \underline{r}} \leq|i| \leq q+\frac{\log \underline{r}}{\log \rho} .
$$

We shall use the same notation $\sigma: \Lambda_{q}^{\mathbb{N}} \rightarrow \Lambda_{q}^{\mathbb{N}}$ to denote the left-shift operator according to $\Lambda_{q}$. Let $\mathcal{I}_{q}=\left\{f_{i}\right\}_{i \in \Lambda_{q}}$ be the conformal IFS over $\Lambda_{q}$. By (2.4) we can deduce that the canonical mapping $\Phi_{q}:\left(\Lambda_{q}^{\mathbb{N}}, d_{\rho^{q}}\right) \rightarrow K$ is $C_{2} \cdot R$-Lipschitz, where $R=\operatorname{diam}(V)$. Indeed, for $\underline{i}, \underline{j} \in \Lambda_{q}^{\mathbb{N}}$ with $d_{\rho^{q}}(\underline{i}, \underline{j})=\left(\rho^{q}\right)^{n}$ so that $\left.\underline{i}\right|_{n}=\left.\underline{j}\right|_{n}$, one has

$$
\begin{aligned}
\left|\Phi_{q}(\underline{i})-\Phi_{q}(\underline{j})\right| & \leq C_{2} \cdot \bar{r}_{i_{1} \cdots i_{n}} \cdot\left|x_{\sigma^{n} \underline{i}}-x_{\sigma^{n} \underline{j}}\right| \\
& \leq C_{2} R \cdot \bar{r}_{i_{1} \cdots i_{n}} \\
& \leq C_{2} R \cdot\left(\rho^{q}\right)^{n} \\
& =C_{2} R \cdot d_{\rho^{q}}(\underline{i}, \underline{j}) .
\end{aligned}
$$

We consider the Gibbs measure $\mu_{q}$ on $\Lambda_{q}^{\mathbb{N}}$ with respect to the potential $\varphi$. Observe that it is the same Gibbs measure as $\mu$ on embedding $\Lambda_{q}^{\mathbb{N}}$ into $\Lambda^{\mathbb{N}}$. To show that the compact group extension $\left(\Lambda_{q}^{\mathbb{N}} \times G, \mathcal{B} \otimes \mathcal{B}_{G}, \mu_{q} \times \xi, \sigma_{\phi_{q}}\right)$ is also ergodic, where

$$
\phi_{q}(\underline{i})=O_{i_{1}}(\Phi(\sigma \underline{i})) \text { for } \underline{i}=i_{1} i_{2} \cdots \in \Lambda_{q}^{\mathbb{N}}
$$

and $\sigma_{\phi_{q}}$ is the skew product of $\phi_{q}$ with respect to the left shift $\sigma$ on $\Lambda_{q}$, we need the following lemma.

Lemma 3.3. Under (A2), $\sigma_{\phi_{q}}$ has a dense orbit in $\Lambda_{q}^{\mathbb{N}} \times G$ for each $q \geq 1$.

Proof. Let $\underline{i}=i_{1} i_{2} \cdots \in \Lambda^{\mathbb{N}}$ and $O \in G$ be such that $\left\{\sigma_{\phi}^{n}(\underline{i}, O): n \geq 0\right\}$ is dense in $\Lambda^{\mathbb{N}} \times G$. For $q \geq 1$ denote by $\Lambda_{<q}=\left\{j \in \Lambda^{*}: \bar{r}_{i} \geq \rho^{q}\right\}$. Then $\bigcup_{j \in \Lambda_{<q}}\left\{T_{j} \sigma_{\phi_{q}}^{n}(\underline{i}, O)\right.$ : $n \geq 0\}=\left\{\sigma_{\phi}^{n}(\underline{i}, O): n \geq 0\right\}$ is dense in $\Lambda^{\mathbb{N}} \times G$, where for $j \in \Lambda_{<q}, T_{j} \sigma_{\phi_{q}}^{n}(\underline{i}, O)$ is the unique element in $\Lambda^{\mathbb{N}} \times G$ such that $\sigma_{\phi}^{|j|}\left(T_{j} \sigma_{\phi_{q}}^{n}(\underline{i}, O)\right)=\sigma_{\phi_{q}}^{n}(\underline{i}, O)$. Since $\Lambda_{<q}$ is finite, by Baire's category theorem, there exists a $j \in \Lambda_{<q}$ such that $H_{j}=\overline{\left\{T_{j} \sigma_{\phi_{q}}^{n}(\underline{i}, O): n \geq 0\right\}}$ has non-empty interior in $\Lambda^{\mathbb{N}} \times G$, that is it contains a set $H=[u] \times I$, where $u \in \Lambda^{*}$ is finite word and $I \subset G$ has non-empty interior. Take an element $g$ in the interior of $I$, then $\sigma^{|u|} H g^{-1}=\left\{\left(\sigma^{|u|} \underline{k}, g^{-1} h\right):(\underline{k}, h) \in H\right\}$ contains the full set $\Lambda^{\mathbb{N}}$ times a set $V^{\prime}$ containing a neighbourhood of the identity in $G$. Since a closed group generated by a set of elements coincides with the closed semigroup generated by them and a compact connected Lie group is generated by any neighbourhood of its identity, we have $\sigma^{|u|} \mathrm{Hg}^{-1}=\Lambda^{\mathbb{N}} \times G$.

Let $n_{q}=\min \left\{|i|: i \in \Lambda_{q}\right\}$. By (3.3) one has $n_{q} \rightarrow \infty$ as $q \rightarrow \infty$. By (2.7) we have for $i, j \in \Lambda_{q}^{*}$,

$$
c_{q}^{-1} \mu_{q}([i]) \mu_{q}([j]) \leq \mu_{q}([i j]) \leq c_{q} \mu_{q}([i]) \mu_{q}([j]),
$$

where $c_{q}=e^{\kappa \frac{\beta^{n q}}{1-\beta}+\kappa \beta^{n_{q}}}$. This is due to the fact that for $i, j \in \Lambda_{q}^{*}$, if either $i$ or $j$ is the empty word $\emptyset$ then we have $\mu_{q}([i j])=\mu_{q}([i]) \mu_{q}([j])$ and if both $i, j \neq \emptyset$ then $\min \{|i|,|j|\} \mid \geq n_{q}$. 
Since $n_{q} \rightarrow \infty$ as $q \rightarrow \infty$, one has $c_{q} \rightarrow 1$ as $q \rightarrow \infty$. For a measure $\nu$ and a measurable set $B$ with $\nu(B)>0$ denote by

$$
\nu_{B}=\left.\frac{1}{\nu(B)} \nu\right|_{B}
$$

In particular for $\nu=\mu_{q}$ we shall use the notation $\mu_{q, B}:=\left(\mu_{q}\right)_{B}$. By (3.4) we have for $n \geq 1$ and $i \in \Lambda_{q}^{n}$ that

$$
c_{q}^{-1}\left(\sigma^{n} \mu_{q}\right)_{[i]} \leq \mu_{q} \leq c_{q}\left(\sigma^{n} \mu_{q}\right)_{[i]}
$$

since for any $j \in \Lambda^{*}$,

$$
\begin{aligned}
\left(\sigma^{n} \mu_{q}\right)_{[i]}([j]) & =\left.\frac{1}{\sigma^{n} \mu_{q}([i])}\left(\sigma^{n} \mu_{q}\right)\right|_{[i]}([j]) \\
& =\frac{1}{\mu_{q}([i])} \sum_{k_{1} \cdots k_{n} \in \Lambda_{q}^{n}} \mu_{q}\left(\left[k_{1} \cdots k_{n} j\right] \cap[i]\right) \\
& =\frac{1}{\mu_{q}([i])} \mu_{q}([i j]) .
\end{aligned}
$$

As before, $\Pi_{d, k}$ is the set of orthogonal projections from $\mathbb{R}^{d}$ onto its $k$-dimensional subspaces, and $G=S O(d, \mathbb{R})$ is the rotation group. We shall need the following Lemma.

Lemma 3.4. For all $q \geq 1, \pi \in \Pi_{d, k}, O \in G, \underline{i} \in \Lambda_{q}^{\mathbb{N}}$ and $n \geq 1$,

$$
H_{C_{1}^{2} \rho^{q}}\left(\pi O \cdot O_{\left.\underline{i}\right|_{n}}\left(x_{\sigma^{n} \underline{i}}\right) \Phi_{q} \mu_{q}\right) \leq \log c_{q}+c_{q} H_{\left(\underline{r} \rho^{q}\right)^{n+1}}\left(\pi O \Phi_{q} \mu_{q,\left[\left.\underline{i}\right|_{n}\right]}\right) .
$$

Proof. Recall that

$$
H_{r}(\nu)=\int_{\operatorname{supp}(\nu)}-\log \nu(B(x, r)) \nu(d x) .
$$

Shortly denote by $g=\pi O \cdot O_{\left.\underline{i}\right|_{n}}\left(x_{\sigma^{n} \underline{i}}\right) \Phi_{q}$. By (3.5) we have

$$
\begin{aligned}
& H_{C_{1}^{2} \rho^{q}}\left(g \mu_{q}\right) \\
= & \int_{\Lambda_{q}^{\mathbb{N}}}-\log \mu_{q}\left(\left\{\underline{j} \in \Lambda_{q}^{\mathbb{N}}:|g(\underline{j})-g(\underline{k})| \leq C_{1}^{2} \rho^{q}\right\}\right) \mu_{q}(d \underline{k}) \\
\leq & \log c_{q}+c_{q} \int_{\Lambda_{q}^{\mathbb{N}}}-\log \left(\sigma^{n} \mu_{q}\right)_{\left[\left.\underline{i}\right|_{n}\right]}\left(\left\{\underline{j} \in \Lambda_{q}^{\mathbb{N}}:|g(\underline{j})-g(\underline{k})| \leq C_{1}^{2} \rho^{q}\right\}\right)\left(\sigma^{n} \mu_{q}\right)_{\left[\left.\underline{i}\right|_{n}\right]}(d \underline{k}) \\
= & \log c_{q}+c_{q} \int_{\Lambda_{q}^{\mathbb{N}}}-\log \mu_{q,\left[\left.\underline{i}\right|_{n}\right]}\left(\left\{\underline{j} \in \Lambda_{q}^{\mathbb{N}}:\left|g\left(\sigma^{n} \underline{j}\right)-g\left(\sigma^{n} \underline{k}\right)\right| \leq C_{1}^{2} \rho^{q}\right\}\right) \mu_{q,\left[\left.\underline{i}\right|_{n}\right]}(d \underline{k}) .
\end{aligned}
$$

We claim that for each $\underline{j}, \underline{k} \in\left[\left.\underline{i}\right|_{n}\right]$, if

$$
\left|\pi O x_{\underline{j}}-\pi O x_{\underline{k}}\right| \leq \bar{r}_{\left.\underline{i}\right|_{n}} \rho^{q},
$$

then

$$
\left|g\left(\sigma^{n} \underline{j}\right)-g\left(\sigma^{n} \underline{k}\right)\right| \leq C_{1}^{2} \rho^{q} .
$$

Since, by (3.2),

$$
\bar{r}_{\left.\underline{i}\right|_{n}} \rho^{q} \geq\left(\underline{r} \rho^{q}\right)^{n} \rho^{q} \geq\left(\underline{r} \rho^{q}\right)^{n+1}
$$

this implies that

$$
\left\{\underline{j} \in \Lambda_{q}^{\mathbb{N}}:\left|\pi O x_{\underline{j}}-\pi O x_{\underline{k}}\right| \leq\left(\underline{r} \rho^{q}\right)^{n+1}\right\} \subset\left\{\underline{j} \in \Lambda_{q}^{\mathbb{N}}:\left|g\left(\sigma^{n} \underline{j}\right)-g\left(\sigma^{n} \underline{k}\right)\right| \leq C_{1}^{2} \rho^{q}\right\} .
$$


Then by (3.6) we may deduce that

$$
\begin{aligned}
& H_{C_{1}^{2} \rho^{q}}\left(g \mu_{q}\right) \\
\leq & \log c_{q}+c_{q} \int_{\Lambda_{q}^{\mathbb{N}}}-\log \mu_{q,\left[\underline{\left.\right|_{i}} n\right]}\left(\left\{\underline{j} \in \Lambda_{q}^{\mathbb{N}}:\left|\pi O x_{\underline{j}}-\pi O x_{\underline{k}}\right| \leq\left(\underline{r} \rho^{q}\right)^{n+1}\right\}\right) \mu_{q,\left[\left.\underline{i}\right|_{n}\right]}(d \underline{k}) \\
= & \log c_{q}+c_{q} H_{\left(\underline{r} \rho^{q}\right)^{n+1}}\left(\pi O \Phi_{q} \mu_{q,[\underline{\underline{k}} n]}\right) .
\end{aligned}
$$

Now we prove our claim. Fix $\underline{j}, \underline{k} \in\left[\left.\underline{i}\right|_{n}\right]$. For $r>0$ write $S_{r}(x)=r\left(x-x_{\sigma^{n} \underline{\underline{i}}}\right)+x_{\sigma^{n} \underline{i}}$ for $x \in V$. Note that $\left|S_{r}\left(x_{\sigma^{n} \underline{j}}\right)-S_{r}\left(x_{\sigma^{n} \underline{k}}\right)\right| \leq R \cdot r$ as well as

$$
\left|S_{r}\left(x_{\sigma^{n} \underline{j}}\right)-x_{\sigma^{n} \underline{\underline{i}}}\right| \vee\left|S_{r}\left(x_{\sigma^{n} \underline{k}}\right)-x_{\sigma^{n} \underline{i}}\right| \leq R \cdot r,
$$

where we recall that $R=\operatorname{diam}(V)$. First, by Lemma 3.2 and the fact that $S_{r}$ only consists of scaling and translation, we have

$$
\begin{aligned}
& \left|\pi O f_{\left.\underline{i}\right|_{n}}\left(S_{r}\left(x_{\sigma^{n} \underline{j}}\right)\right)-\pi O f_{\left.\underline{i}\right|_{n}}\left(S_{r}\left(x_{\sigma^{n} \underline{k}}\right)\right)\right| \\
& \leq \bar{r}_{\left.\underline{i}\right|_{n}}\left|\pi O \cdot O_{\left.\underline{i}\right|_{n}}\left(x_{\sigma^{n} \underline{i}}\right)\left(S_{r}\left(x_{\sigma^{n} \underline{j}}\right)-S_{r}\left(x_{\sigma^{n} \underline{k}}\right)\right)\right|+2 C_{3} R^{2} r^{2} \bar{r}_{\left.\underline{i}\right|_{n}} \\
& =r\left(\bar{r}_{\left.\underline{i}\right|_{n}}\left|\pi O \cdot O_{\left.\underline{i}\right|_{n}}\left(x_{\sigma^{n} \underline{\underline{i}}}\right)\left(x_{\sigma^{n} \underline{j}}-x_{\sigma^{n} \underline{k}}\right)\right|+2 C_{3} R^{2} r \bar{r}_{\left.\underline{i}\right|_{n}}\right) \\
& \leq r\left(C_{1}\left|\pi O f_{\left.\underline{i}\right|_{n}}\left(x_{\sigma^{n} \underline{j}}\right)-\pi O f_{\left.\underline{\underline{i}}\right|_{n}}\left(x_{\sigma^{n} \underline{k}}\right)\right|+2\left(C_{1}+1\right) C_{3} R^{2} r \bar{r}_{\underline{i}_{n}}\right) \text {. }
\end{aligned}
$$

This implies that if

$$
\left|\pi O x_{\underline{j}}-\pi O x_{\underline{k}}\right|=\left|\pi O f_{\left.\underline{\underline{i}}\right|_{n}}\left(x_{\sigma^{n} \underline{j}}\right)-\pi O f_{\left.\underline{i}\right|_{n}}\left(x_{\sigma^{n} \underline{k}}\right)\right| \leq \bar{r}_{\left.\underline{i}\right|_{n}} \rho^{q},
$$

then for any $\epsilon>0$, for all $r \in\left(0, \frac{\epsilon \rho^{q}}{2\left(C_{1}+1\right) C_{3} R^{2}}\right)$,

$$
\left|\pi O f_{\left.\underline{\underline{i}}\right|_{n}}\left(S_{r}\left(x_{\sigma^{n} \underline{j}}\right)\right)-\pi O f_{\left.\underline{\underline{\left.\right|_{1}}}\right|_{n}}\left(S_{r}\left(x_{\sigma^{n} \underline{k}}\right)\right)\right| \leq\left(C_{1}+\epsilon\right) r \bar{r}_{\underline{\underline{i}} n} \rho^{q} .
$$

On the other hand, note that

$$
r\left(g\left(\sigma^{n} \underline{j}\right)-g\left(\sigma^{n} \underline{k}\right)\right)=\pi O \cdot O_{\left.\underline{i}\right|_{n}}\left(x_{\sigma^{n} \underline{i}}\right)\left(S_{r}\left(x_{\sigma^{n} \underline{j}}\right)-S_{r}\left(x_{\sigma^{n} \underline{k}}\right)\right) .
$$

Thus, by Lemma 3.2 again, for all $r \in\left(0, \frac{\epsilon \rho^{q}}{2\left(C_{1}+1\right) C_{3} R^{2}}\right)$,

$$
r\left|g\left(\sigma^{n} \underline{j}\right)-g\left(\sigma^{n} \underline{k}\right)\right| \leq C_{1}\left(\left(C_{1}+\epsilon\right) r \rho^{q}+2 C_{3} R^{2} r^{2}\right),
$$

which implies that

$$
\left|g\left(\sigma^{n} \underline{j}\right)-g\left(\sigma^{n} \underline{k}\right)\right| \leq C_{1}\left(\left(C_{1}+\epsilon\right) \rho^{q}+2 C_{3} R^{2} r\right) .
$$

This yields that

$$
\left|g\left(\sigma^{n} \underline{j}\right)-g\left(\sigma^{n} \underline{k}\right)\right| \leq C_{1}\left(C_{1}+\epsilon\right) \rho^{q}
$$

holds for all $\epsilon>0$, therefore

$$
\left|g\left(\sigma^{n} \underline{j}\right)-g\left(\sigma^{n} \underline{k}\right)\right| \leq C_{1}^{2} \rho^{q} .
$$

For $\pi \in \Pi_{d, k}, q \in \mathbb{N}$ and $\nu$ a measure on $\mathbb{R}^{d}$, define

$$
e_{q}(\pi, \nu)=\frac{1}{-\log C_{1}^{2}+q \log (1 / \rho)} H_{C_{1}^{2} \rho^{q}}(\pi \nu) .
$$

So $e_{q}: \Pi_{d, k} \times \mathcal{M} \rightarrow[0, k]$ is lower semicontinuous. Define

$$
E_{q}(\pi)=\mathbb{E}_{\mu_{q} \times \xi}\left(e_{q}\left(\pi, O \Phi_{q} \mu_{q}\right)\right) .
$$


Proposition 3.5. For all $q \geq 1$, for $\xi$-a.e. $O \in G$ and $\mu_{q}$-a.e. $\underline{i} \in \Lambda_{q}^{\mathbb{N}}$,

$$
\liminf _{N \rightarrow \infty} \frac{1}{N} \sum_{n=1}^{N} H_{\left(\underline{r} \rho^{q}\right)^{n+1}}\left(\pi O \Phi_{q} \mu_{q,\left[\left.\underline{i}\right|_{n}\right]}\right) \geq \frac{-\log C_{1}^{2}+q \log (1 / \rho)}{c_{q}} \cdot E_{q}(\pi)-\frac{\log c_{q}}{c_{q}},
$$

for all $\pi \in \Pi_{d, k}$.

Proof. Since $e_{q}$ is lower semicontinuous, applying Proposition 3.1 under the dynamical system $\left(\Lambda_{q}^{\mathbb{N}} \times G, \mathcal{B} \otimes \mathcal{B}_{G}, \mu_{q} \times \xi, \sigma_{\phi_{q}}\right)$, which is ergodic by Lemma 3.3, to a sequence of continuous functions approximating $e_{q}$ from below and using the monotone convergence theorem, we have that for $\xi$-a.e. $O$ and $\mu_{q}$-a.e. $\underline{i}$,

$$
\lim _{N \rightarrow \infty} \inf \frac{1}{N} \sum_{n=0}^{N-1} e_{q}\left(\pi, O \cdot O_{\left.\underline{i}\right|_{n}}\left(x_{\sigma^{n} \underline{i}}\right) \Phi_{q} \mu_{q}\right) \geq E_{q}(\pi) \text { for all } \pi \in \Pi_{d, k} .
$$

Hence, by Lemma 3.4, for all $q \geq 1$, for $\xi$-a.e. $O \in G$ and $\mu_{q}$-a.e. $\underline{i} \in \Lambda_{q}^{\mathbb{N}}$,

$$
\left.\frac{1}{-\log C_{1}^{2}+q \log (1 / \rho)}\left[\log c_{q}+c_{q} \liminf _{N \rightarrow \infty} \frac{1}{N} \sum_{n=1}^{N} H_{\left(\underline{\left.\underline{r} \rho^{q}\right)^{n+1}}\right.}\left(\pi O \Phi_{q} \mu_{q,[\underline{\underline{i}} n]}\right]\right)\right] \geq E_{q}(\pi)
$$

for all $\pi \in \Pi_{d, k}$, which yields the conclusion.

Theorem 3.6. We have for all $q \geq 1$, for $\xi$-a.e. $O \in G$,

$$
\operatorname{dim}_{H}(\pi O \Phi \mu) \geq \frac{-\log C_{1}^{2}+q \log (1 / \rho)}{c_{q}(q \log (1 / \rho)-\log \underline{r})} E_{q}(\pi)-\frac{\log C^{\prime}}{q \log (1 / \rho)-\log \underline{\underline{r}}}-\frac{\log c_{q}}{c_{q}(q \log (1 / \rho)-\log \underline{r})}
$$

for all $\pi \in \Pi_{d, k}$, where $C^{\prime}$ is a constant depending only on $C_{2} \cdot R$, and $c_{q}$ is given in (3.4).

Proof. First we pick a $\xi$-typical $O \in G$ such that the statement of Proposition 3.5 holds. The mapping $f \equiv \pi O \Phi_{q}:\left(\Lambda_{q}^{\mathbb{N}}, d_{\rho^{q}}\right) \rightarrow \mathbb{R}$ is $C_{2} \cdot R$-Lipschitz. By [12, Theorem 5.4], there exist a $\rho^{q}$-tree $\left(X, d_{\rho^{q}}\right)$ and maps $\Lambda_{q}^{\mathbb{N}} \stackrel{h}{\rightarrow} X \stackrel{f^{\prime}}{\rightarrow} \mathbb{R}^{k}$ such that $f=f^{\prime} h$, where $h$ is a tree morphism and $f^{\prime}$ is $C$-faithful (see [12, Definition 5.1]) for some constant $C$ depending on only $C_{2} \cdot R$. Then applying [12, Proposition 5.3] to the $\underline{r} \rho^{q}$-tree $\left(X, d_{r \rho^{q}}\right)$ (for which $f^{\prime}$ is $\underline{r}^{-1} C$-faithful), there is a $C^{\prime}$ depending only on $\underline{r}^{-1} C$ such that for all $n \geq 1$,

$$
\left|H_{\left(\underline{r} \rho^{q}\right)^{n+1}}\left(f \mu_{q,\left[\left.\underline{i}\right|_{n}\right]}\right)-H_{\left(\underline{r} \rho^{q}\right)^{n+1}}\left(h \mu_{q,\left[\left.\underline{i}\right|_{n}\right]}\right)\right| \leq C^{\prime} .
$$

Then, using Proposition 3.5 , for $\mu_{q}$-a.e. $\underline{i}$,

$$
\begin{aligned}
\frac{1}{-\log C_{1}^{2}+q \log (1 / \rho)} & \liminf _{N \rightarrow \infty} \frac{1}{N} \sum_{n=1}^{N} H_{\left(\underline{r} \rho^{q}\right)^{n+1}}\left(h \mu_{q,\left[\left.\underline{i}\right|_{n}\right]}\right) \\
& \geq \frac{1}{c_{q}} E_{q}(\pi)-\frac{\log C^{\prime}}{-\log C_{1}^{2}+q \log (1 / \rho)}-\frac{\log c_{q}}{c_{q}\left(-\log C_{1}^{2}+q \log (1 / \rho)\right)}
\end{aligned}
$$

for all $\pi \in \Pi_{d, k}$. Now, using [12, Theorem 4.4], it follows that

$$
\operatorname{dim}_{H} h \mu_{q} \geq \frac{-\log C_{1}^{2}+q \log (1 / \rho)}{c_{q}(q \log (1 / \rho)-\log \underline{r})} E_{q}(\pi)-\frac{\log C^{\prime}}{q \log (1 / \rho)-\log \underline{r}}-\frac{\log c_{q}}{c_{q}(q \log (1 / \rho)-\log \underline{r})}
$$

for all $\pi \in \Pi_{d, k}$. Since $f^{\prime}$ is $C$-faithful and $f^{\prime} h \mu_{q}=f \mu_{q}=\pi \Phi_{q} \mu_{q}=\pi \Phi \mu$, the conclusion follows from [12, Proposition 5.2]. 
3.2. Projection theorems. With the approach used in Subsection 3.1, we avoid the need for any separation condition in our projection results. For $\pi \in \Pi_{d, k}$ we have

$$
\begin{aligned}
E_{q}(\pi) & =\mathbb{E}_{\mu_{q} \times \xi}\left(e_{q}\left(\pi, O \Phi_{q} \mu_{q}\right)\right) \\
& =\int_{G} \frac{1}{-\log C_{1}^{2}+q \log (1 / \rho)} H_{C_{1}^{2} \rho^{q}}\left(\pi O \Phi_{q} \mu_{q}\right) \xi(d O) \\
& =\int_{G} \frac{1}{-\log C_{1}^{2}+q \log (1 / \rho)} H_{C_{1}^{2} \rho^{q}}(\pi O \Phi \mu) \xi(d O),
\end{aligned}
$$

where we have used the fact that $\Phi_{q} \mu_{q}=\Phi \mu$ for all $q \geq 1$.

Theorem 3.7. The limit

$$
E(\pi):=\lim _{q \rightarrow \infty} E_{q}(\pi)
$$

exists for every $\pi \in \Pi_{d, k}$. Moreover:

(i) For a fixed $\pi \in \Pi_{d, k}$, for $\xi$-a.e. $O$,

$$
\operatorname{dim}_{e} \pi O \Phi \mu=\operatorname{dim}_{H} \pi O \Phi \mu=E(\pi) .
$$

(ii) For $\xi$-a.e. $O$,

$$
\operatorname{dim}_{H} \pi O \Phi \mu \geq E(\pi) \quad \text { for all } \pi \in \Pi_{d, k} .
$$

Proof. For all integers $q \geq 1$, by Theorem 3.6 we have that for $\xi$-a.e. $O \in G$, $\operatorname{dim}_{H}(\pi O \Phi \mu) \geq \frac{-\log C_{1}^{2}+q \log (1 / \rho)}{c_{q}(q \log (1 / \rho)-\log \underline{r})} E_{q}(\pi)-\frac{\log C^{\prime}}{q \log (1 / \rho)-\log \underline{\underline{r}}}-\frac{\log c_{q}}{c_{q}(q \log (1 / \rho)-\log \underline{r})}$ for all $\pi \in \Pi_{d, k}$. As integers $\{q \geq 1\}$ are countable, we may take a $\xi$-full set such that the above statement is true for all $q \geq 1$. Since $C_{1}, C^{\prime}$ and $\underline{r}$ do not depend on $q$ and $c_{q} \rightarrow 1$ as $q \rightarrow \infty$, we obtain for $\xi$-a.e. $O \in G$,

$$
\operatorname{dim}_{H}(\pi \Phi O \mu) \geq \limsup _{q \rightarrow \infty} E_{q}(\pi)
$$

for all $\pi \in \Pi_{d, k}$. On the other hand, we also know that $\operatorname{dim}_{e}(\nu) \geq \operatorname{dim}_{H}(\nu)$ for any Borel probability measure $\nu$. Thus, applying Fatou's lemma to (3.10), we have

$$
\begin{aligned}
\limsup _{q \rightarrow \infty} E_{q}(\pi) & \leq \mathbb{E}_{\xi}\left(\operatorname{dim}_{H}(\pi O \Phi \mu)\right) \\
& \leq \mathbb{E}_{\xi}\left(\operatorname{dim}_{e}(\pi O \Phi \mu)\right) \\
& \leq \liminf _{q \rightarrow \infty} E_{q}(\pi) .
\end{aligned}
$$

This shows that $\lim _{q \rightarrow \infty} E_{q}(\pi)$ exists for all $\pi \in \Pi_{d, k}$, and (i) and (ii) follow directly.

Write $\beta=\min \left\{k, \operatorname{dim}_{H} \Phi \mu\right\}$.

Corollary 3.8. (i) $E(\pi)=\beta$ for all $\pi \in \Pi_{d, k}$; (ii) $\operatorname{dim}_{H}(\pi \Phi \mu)=\beta$ for all $\pi \in \Pi_{d, k}$.

Proof. (i) For a fixed $\pi \in \Pi_{d, k}$, since $\Phi \mu$ is exact-dimensional (see [9]), using Theorem 3.7 (i) and applying the Marstrand's projection theorem for measures (see [13]), for $\xi$-a.e. $O \in S O(d, \mathbb{R})$,

$$
E(\pi)=\operatorname{dim}_{H} \pi O \Phi \mu=\min \left\{k, \operatorname{dim}_{H} \Phi \mu\right\}=\beta .
$$

This implies that $E(\pi)=\beta$ for all $\pi \in \Pi_{d, k}$.

(ii) By (i) and Theorem 3.7 (ii), we have for $\xi$-a.e. $O \in S O(d, \mathbb{R})$,

$$
\operatorname{dim}_{H} \pi O \Phi \mu \geq \beta \quad \text { for all } \pi \in \Pi_{d, k} \text {. }
$$

Then we get the conclusion from the fact that $\beta \geq \operatorname{dim}_{H} \pi O \Phi \mu$ for any $\pi \in \Pi_{d, k}$ and $O \in S O(d, \mathbb{R})$. 
For $C^{1}$-images, we need the following Proposition:

Proposition 3.9. Let $\pi \in \Pi_{d, k}$. For all $C^{1}$-maps $h: K \mapsto \mathbb{R}^{k}$ such that $\sup _{x \in K} \| D_{x} h-$ $\pi \|<c \rho^{q}$, we have that for all $q \geq 1$, for $\xi$-almost every $O$,

$$
\begin{aligned}
& \operatorname{dim}_{H} h O \Phi \mu \geq \\
& \quad \frac{-\log C_{1}^{2}+q \log (1 / \rho)}{c_{q}(q \log (1 / \rho)-\log \underline{r})} E_{q}(\pi)-\frac{\log C^{\prime}}{q \log (1 / \rho)-\log \underline{r}}-\frac{\log c_{q}}{c_{q}(q \log (1 / \rho)-\log \underline{r})}-O(1 / q),
\end{aligned}
$$

where the constant $O(1 / q)$ only depends on $\rho, c$ and $k$.

Proof. The proof is similar to that of [12, Proposition 8.4] together with Proposition 3.5 and Theorem 3.6 .

Corollary 3.8 and Proposition 3.9 imply the following.

Corollary 3.10. For all $C^{1}$-maps $h: K \rightarrow \mathbb{R}^{k}$ without singular points,

$$
\operatorname{dim}_{H} h \Phi \mu=\min \left\{k, \operatorname{dim}_{H} \Phi \mu\right\} .
$$

Proof. Since $h$ is a $C^{1}-\operatorname{map}, \operatorname{dim}_{H} h O \Phi \mu \leq \min \left\{k, \operatorname{dim}_{H} \Phi \mu\right\}$. The lower bound follows from Proposition 3.9 applied to the restricted measures $\left.\mu\right|_{[\underline{\underline{i}} \mid n]}$ for $\underline{i} \in \Lambda^{\mathbb{N}}$ and $n \geq 1$ sufficiently large.

\section{ACKNOWLEDGEMENTS}

We would like to thank Julien Barral, Kenneth Falconer, Jonathan Fraser and Thomas Jordan for helpful discussions and comments. Part of this work was completed while the second author was visiting the Institut Mittag-Leffler as part of the 'Fractal Geometry and Dynamics' Programme, he would like to thank the organisers and staff members for their hospitality.

\section{REFERENCES}

[1] T. Bedford and A. M. Fisher, Ratio geometry, rigidity and the scenery process for hyperbolic Cantor sets. Ergodic Theory Dynam. Systems 17: 531-564, 1997.

[2] T. Bedford, A. M. Fisher, M. Urbaski The scenery flow for hyperbolic Julia sets. Proceedings of the London Mathematical Society, 85(2): 467-492, 2002.

[3] R. Bowen. Equilibrium states and the ergodic theory of Anosov diffeomorphisms. Vol 470. Berlin: Springer-Verlag, 1975.

[4] R. Bowen. Hausdorff dimension of quasicircles. Publ. math. IHES, 50(1): 11-25, 1979.

[5] K Falconer. Fractal Geometry: Mathematical Foundations and Applications. 3rd Edition. Chichester: Wiley. 2014.

[6] K. Falconer, J. Fraser and X. Jin. Sixty years of fractal projections. Fractal geometry and stochastics $V$. Birkhuser, Cham, 3-25, 2015.

[7] K. Falconer and X. Jin. Exact dimensionality and projections of random self-similar measures and sets. J. London Math. Soc. (2) 90: 388-412, 2014.

[8] A.-H. Fan, K.-S. Lau and H. Rao. Relationships between different dimensions of a measure. Monatshefte fr Mathematik 135(3): 191-201, 2002.

[9] D.-J. Feng and H. Hu. Dimension theory of iterated function systems. Comm. Pure Appl. Math. 62: $1435-1500,2009$.

[10] J. M. Fraser and M. Pollicott. Micromeasure distributions and applications for conformally generated fractals. Mathematical Proceedings of the Cambridge Philosophical Society. Vol. 159. No. 03., Cambridge University Press, 2015.

[11] H. Furstenberg. Ergodic fractal measures and dimension conservation. Ergod. Th. 83 Dynam. Sys. 28: 405-422, 2008.

[12] M. Hochman and P. Shmerkin. Local entropy averages and projections of fractal measures. Ann. of Math. (2) 175: 1001-1059, 2012.

[13] B. R. Hunt and V. Y. Kaloshin. How projections affect the dimension spectrum of fractal measures. Nonlinearity 10(5): 1031, 1997. 
[14] J. M. Marstrand. Some fundamental geometrical properties of plane sets of fractional dimensions. Proceedings of the London Math. Soc. 3.1: 257-302, 1954.

[15] P. Mattila. Haudsorff dimension, orthogonal projections and intersections with planes. Ann. Acad. Sci. Fenn. Ser. A I Math. 1: 227-244, 1975.

[16] P. Mattila. Hausdorff dimension, projections, intersections, and Besicovitch sets. arXiv preprint arXiv:1712.09199. 2017.

[17] W. Parry Skew products of shifts with a compact Lie group. J.LondonMath.Soc. (2):56, 395-404, 1997.

[18] N. Patzschke Self-conformal multifractal measures. Adv. in Appl. Math. 19: 486-513, 1997.

[19] Y. Peres, M. Rams, K. Simon and B. Solomyak. Equivalence of positive Hausdorff measure and the open set condition for self-conformal sets. Proceedings of the American Mathematical Society 129(9): 2689-2699, 2001.

[20] D. Ruelle. Thermodynamic Formalism, 2nd edn. New York: Cambridge University Press. 2004.

[21] P. Shmerkin. Projections of self-similar and related fractals: a survey of recent developments. Fractal Geometry and Stochastics V. Birkhuser, Cham, 53-74, 2015.

[22] P. Shmerkin. On the Hausdorff dimension of pinned distance sets. arXiv:1706.00131, 2018.

[23] S. Silverman. On maps with dense orbits and the definition of chaos. The Rocky Mountain Journal of Mathematics. 22.1: 353-75, 1992.

[24] D. Sullivan. Differentiable structures on fractal-like sets, determined by intrinsic scaling functions on dual Cantor sets, in The Mathematical Heritage of Hermann Weyl (Durham, NC, 1987), Proc. Sympos. Pure Math. 48, Amer. Math. Soc., Providence, RI, 15-23, 1988.

School of Mathematics, University of Manchester, Oxford Road, Manchester M13 9PL, UNITED KINGDOM

E-mail address: catherine.bruce@manchester.ac.uk

School of Mathematics, University of Manchester, Oxford Road, Manchester M13 9PL, UNITED KINGDOM

E-mail address: xiong.jin@manchester.ac.uk 